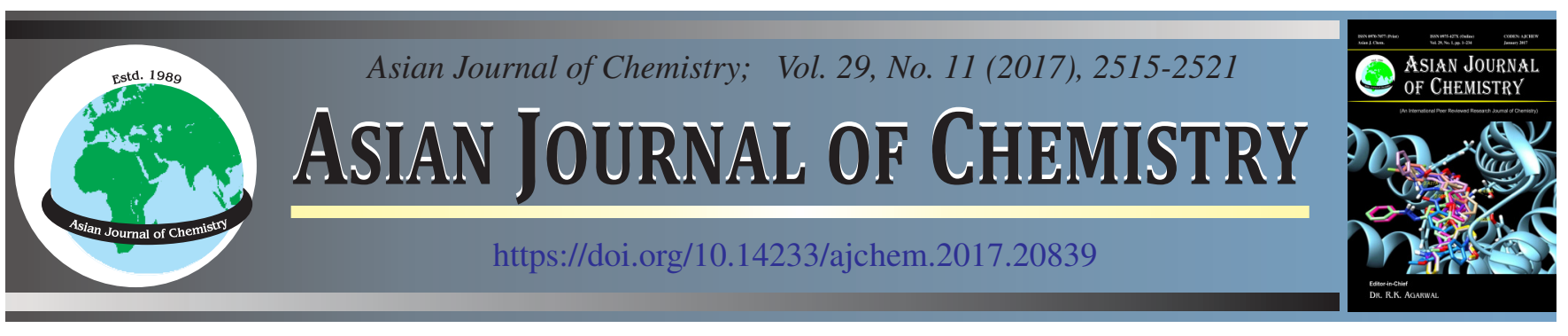

\title{
DFT-Based Prediction of Bioconcentration Factors of Polychlorinated Biphenyls in Fish Species Using Atomic Descriptors
}

\author{
Anil Kumar Soni ${ }^{1}$, Vishnu Kumar Sahu ${ }^{2}$ and Sangeeta Sahu ${ }^{3, *}$
}

\author{
${ }^{1}$ Department of Chemistry, Shia P.G. College, Lucknow-226030, India \\ ${ }^{2}$ Department of Chemistry, Maharani Lal Kunwari P.G. College, Balrampur-271 201, India \\ ${ }^{3}$ Department of Chemistry, University of Lucknow, Lucknow-226 007, India \\ *Corresponding author: E-mail: sangeet.chem@gmail.com
}

Received: 3 June 2017;

Accepted: 27 July 2017;

Published online: 29 September 2017;

AJC-18584

\begin{abstract}
Atomic charge and density calculated by DFT method at twelve sites of biphenyl skeleton of polychlorinated biphenyls has been used to predict their bioconcentration factors. For prediction of bioconcentration factor, the 3D modeling and geometry optimization of all the compounds have been performed on workspace program of CAChe pro software of Fujitsu using the B88-PW91 GGA energy function with the DZVP basis set. Bioconcentration factor model, derived from partial atomic charges $\left(r^{2}=0.917, r^{2} \mathrm{Cv}=0.879, \mathrm{~s}=0.270\right)$ has better predicting power than the model derived from HOMO densities $\left(r^{2}=0.902, r^{2} \mathrm{Cv}=0.837, \mathrm{~s}=0.307\right)$ and can be used to predict bioconcentration factors of a large number of related compounds within limited time without any difficulty. One can also use these DFT-based atomic parameters in developing new methodology for chemical degradation of polychlorinated biphenyls.
\end{abstract}

Keywords: Atomic charge, Bioconcentration factor, DFT, HOMO Density, Polychlorinated biphenyls.

\section{INTRODUCTION}

Prediction of bioconcentration factors (BCFs) [1-4] of the polychlorinated biphenyls (PCBs) [5], a group of persistent organic pollutants [6-18] by QSAR modeling [19] using molecular descriptors were made time to time to increase the probability of success and reduce the time and cost in exploring the toxicological and ecological characteristics of molecules [20-35]. In this work, for the first time, we have used atomic descriptors: partial atom charges and HOMO densities [36] at various sites of polychlorinated biphenyls to predict their bioconcentration factors. Our interest in DFT-based prediction of bioconcentration factors using atomic descriptors was stimulated by the fact that DFT methods [37-39] are capable of generating a variety of local reactivity descriptors quite accurately.

\section{EXPERIMENTAL}

Fifty seven polychlorinated biphenyl congeners, listed in Table-1, are the study materials for the present study. These congeners have been taken from the literature with their experimental logarithmic bioconcentration factor values $\left(\log \mathrm{BCF}_{\text {exp }}\right)$ for several fish species [24]. For BCF prediction, the 3D modeling [40] and geometry optimization of all the compounds have been performed on workspace program of CAChe pro software of Fujitsu using the B88-PW91 GGA energy function with the DZVP basis set [41]. The values of descriptors, partial atom charges and HOMO densities at 12 sites of polychlorinated biphenyls, have directly been obtained from DFT calculation results. The Project Leader program associated with CAChe has been used for multiple linear regression (MLR) analysis [42] and various regression equations have been developed for prediction of bioconcentration factor $\left(\log \mathrm{BCF}_{\mathrm{pre}}\right)$.

\section{RESULTS AND DISCUSSION}

The general chemical structure of polychlorinated biphenyls [5] is based upon the biphenyl's skeleton, containing one to ten atoms of chlorine and has 12 sites viz. 1, 2, 3, 4, 5, 6, 1', 2', 3', 4', 5' and 6'(Fig. 1). Biphenyl is well known aromatic compound and like other aromatic compounds its characteristic reaction is also electrophilic substitution reactions. Biphenyl, on chlorination $\left(\mathrm{Fe} / \mathrm{Cl}_{2}\right)$ forms 4-chlorobiphenyl $\left(\Delta \mathrm{H}_{\text {Reaction }}=-28.865 \mathrm{kcal} / \mathrm{mol}, \Delta \mathrm{G}_{\text {Reaction }}=-27.61 \mathrm{kcal} / \mathrm{mol}\right.$ and $\left.\Delta \mathrm{S}_{\text {Reaction }}=-6.839 \mathrm{cal} / \mathrm{mol} \mathrm{K}\right)$ as major product with some 2chlorobiphenyl $\left(\Delta \mathrm{H}_{\text {Reaction }}=-21.335 \mathrm{kcal} / \mathrm{mol}, \Delta \mathrm{G}_{\text {Reaction }}=-20.08\right.$ $\mathrm{kcal} / \mathrm{mol}$ and $\Delta \mathrm{S}_{\text {Reaction }}=-5.529 \mathrm{cal} / \mathrm{mol} \mathrm{K}$ ) as minor product. These on further chlorination give $4,4^{\prime}$-dichlorobiphenyl $\left(\Delta \mathrm{H}_{\text {Reaction }}=-28.238 \mathrm{kcal} / \mathrm{mol}, \Delta \mathrm{G}_{\text {Reaction }}=-25.728 \mathrm{kcal} / \mathrm{mol}\right.$ and $\left.\Delta \mathrm{S}_{\text {Reaction }}=-9.741 \mathrm{cal} / \mathrm{mol} \mathrm{K}\right)$ as major product and some $2,4^{\prime}$ dichlorobiphenyl $\left(\Delta \mathrm{H}_{\text {Reaction }}=-21.335 \mathrm{kcal} / \mathrm{mol}, \Delta \mathrm{G}_{\text {Reaction }}=\right.$ 
TABLE-1

BIPHENYL, ITS CHLORO-DERIVATIVES AND THEIR CONGENERS WITH THEIR EXPERIMENTAL LOGARITHMIC BCF

\begin{tabular}{|c|c|c|c|c|c|}
\hline S. No. & Chemical Name & ${ }^{\mathrm{a}} \log \mathrm{BCF}_{\text {exp }}$ & S. No. & Chemical Name & ${ }^{\mathrm{a}} \log \mathrm{BCF}_{\text {exp }}$ \\
\hline 1 & Biphenyl & 2.64 & 30 & $2,3,3^{\prime}, 4,4^{\prime}$-pentachloro- & 5.00 \\
\hline 2 & 4-chloro- & 2.77 & 31 & 2,3,3 ,4,6-pentachloro- & 5.00 \\
\hline 3 & 2,2`-dichloro- & 3.38 & 32 & $2,3^{`}, 4,4^{\prime}, 5$-pentachloro- & 5.00 \\
\hline 4 & 2,3-dichloro- & 4.11 & 33 & 3,3,4,4,5-pentachloro- & 5.81 \\
\hline 5 & 2,3`-dichloro- & 3.80 & 34 & $2,2^{\prime}, 3,3^{`}, 4,4^{\prime}$-hexachloro- & 5.77 \\
\hline 6 & 2,4-dichloro- & 3.55 & 35 & $2,2 ` 3,3 `, 6,6^{`}$-hexachloro- & 5.43 \\
\hline 7 & 2,4`-dichloro- & 3.57 & 36 & 2,2',3,4,4',5-hexachloro- & 5.88 \\
\hline 8 & 2,5-dichloro- & 3.89 & 37 & $2,2 ` 3,4,4 ` 5$-hexachloro- & 5.39 \\
\hline 9 & 3,5-dichloro- & 3.78 & 38 & $2,2,3,4,5,5^{\prime}$-hexachloro- & 5.81 \\
\hline 10 & 4,4`-dichloro- & 3.28 & 39 & $2,2^{`}, 3,4^{`}, 5,6^{\prime}$-hexachloro- & 5.39 \\
\hline 11 & 2,2,5-trichloro- & 4.11 & 40 & 2,2,3,5,5,6-hexachloro- & 5.54 \\
\hline 12 & 2,4,4`-trichloro- & 4.20 & 41 & $2,2^{\prime}, 4,4^{\prime}, 5,5^{`}$-hexachloro- & 5.65 \\
\hline 13 & 2,4,5-trichloro- & 4.26 & 42 & $2,2^{\prime}, 4,4^{\prime}, 6,6^{\prime}$-hexachloro- & 4.93 \\
\hline 14 & 2,4',5-trichloro- & 4.23 & 43 & 2,3,3,4,4',5-hexachloro- & 5.39 \\
\hline 15 & 2,2'3,3'-tetrachloro- & 4.23 & 44 & 2,3,3'4,4',5'-hexachloro- & 5.39 \\
\hline 17 & 2,2 ,4,4 -tetrachloro- & 4.85 & 46 & $2,2^{\prime}, 3,3^{\prime}, 4,5,6$-heptachloro- & 5.80 \\
\hline 18 & 2,2,4,5-tetrachloro- & 5.00 & 47 & 2,2 ,3,4,4 ,5,5 -heptachloro- & 5.80 \\
\hline 19 & 2,2 ,4,5 -tetrachloro- & 4.84 & 48 & $2,2^{\prime}, 3,4,4 `, 5,6$-heptachloro- & 5.80 \\
\hline 20 & 2,2 ,5,5 -tetrachloro- & 4.63 & 49 & 2,2,3,4,4,5 ,6-heptachloro- & 5.84 \\
\hline 21 & 2,2 ,6,6 -tetrachloro- & 3.85 & 50 & 2,2 ,3,4 ,5,5 ,6-heptachloro- & 5.80 \\
\hline 22 & 2,3,4,6-tetrachloro- & 4.60 & 51 & 2,3,3,4,4,5 ,6-heptachloro- & 5.84 \\
\hline 23 & 2,3',4,5-tetrachloro- & 4.77 & 52 & $2,2^{\prime}, 3,3^{\prime}, 4,4 ` 5,5^{`}$-octachloro- & 5.81 \\
\hline 24 & 3,3 ,4,4`-tetrachloro- & 4.59 & 53 & $2,2,3,3 ` 4,4 ` 5,6$-octachloro- & 5.92 \\
\hline 25 & 2,2`3,4,5 -pentachloro- & 5.38 & 54 & $2,2^{`}, 3,3^{`}, 4,4^{`} 5,6^{`}$-octachloro- & 5.92 \\
\hline 26 & 2,2 ,3,4,5-pentachloro- & 5.00 & 55 & 2,2`,3,3`4, 5,5 ,6-octachloro- & 5.88 \\
\hline 27 & 2,2`,3`,4,5 -pentachloro- & 5.43 & 56 & $2,2^{\prime}, 3,3^{\prime}, 5,5^{`}, 6,6^{`}$-octachloro- & 5.82 \\
\hline 28 & 2,2 ,4,4,5-pentachloro- & 5.00 & 57 & $2,2^{\prime}, 3,3^{`}, 4,5,5^{\prime}, 6,6^{\prime}$-nonachloro- & 5.71 \\
\hline 29 & $2,2^{`}, 4,5,5^{`}$-pentachloro- & 5.40 & 58 & Decachloro- & 5.44 \\
\hline
\end{tabular}

${ }^{a}$ Experimental logarithmic BCF values as taken from literature [Ref. 24]

$-19.453 \mathrm{kcal} / \mathrm{mol}$ and $\left.\Delta \mathrm{S}_{\text {Reaction }}=-6.954 \mathrm{cal} / \mathrm{mol} \mathrm{K}\right)$ and $2,2^{\prime}-$ dichlorobiphenyl $\left(\Delta \mathrm{H}_{\text {Reaction }}=-6.275 \mathrm{kcal} / \mathrm{mol}, \Delta \mathrm{G}_{\text {Reaction }}=\right.$ $-3.765 \mathrm{kcal} / \mathrm{mol}$ and $\Delta \mathrm{S}_{\text {Reaction }}=-8.978 \mathrm{cal} / \mathrm{mol} \mathrm{K}$ ), respectively [43]. The vibrational frequency calculations were performed to determine the above thermodynamic quantities at $298 \mathrm{~K}$ by DFT method. Actually in biphenyl the presence of one phenyl ring is found to activate the other for electrophilic substitution and directs the new group to $o$ - and $p$-positions. In monosubstituted biphenyl, the substituent invariably enters the unsubstituted ring (in $o$ - and $p$-positions) whether the substituent already present is activating or deactivating. In order to predict the electrophilic sites of attack, most negative charged sites $\left(\mathrm{Q}_{\min }\right)$ and/or orbital of high electron density (HOMO) must be known [44,45]. The partial atomic charges and HOMO densities of all the twelve sites have been evaluated by DFT method and are reported in Tables 2 and 3, respectively.

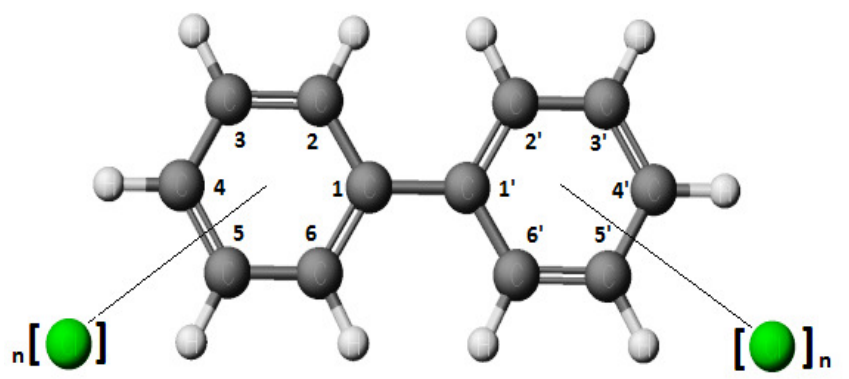

Fig. 1
To correlate the effect of the number and position of chlorine atoms on atomic charges and HOMO densities at these sites and finally their effect on the bioconcentration factors, QSAR models have been developed. For sake of simplicity, the study has been divided into following sub-headings:

Partial atomic charge $(\mathrm{Q})$ : The charge distributions $(\mathrm{Q})$ of each atom [44] of all the polychlorinated biphenyls were calculated by DFT method and the results are reported in Table-2. A reference to this table shows that all the compounds bear positive charges on sites 1 and 1' indicating that the rest sites are suitable for the attack of $\mathrm{Cl}^{+}$ion (an electrophile) for substitution reaction. MLR analyses [42] were employed to develop reliable QSAR model for the prediction of bioconcentration factor. For MLR analysis, partial atomic charges of 12 sites (Table-2) were used as independent variables and the experimental logarithmic bioconcentration factor values as dependent variable. MLR analysis has been made by Project Leader software associated with CAChe, using these charges in various combinations. The best twelve models for $\mathrm{k}=1,2$, $3,4,5,6,7,8,9,10,11$ and 12 (eqns. 1-12) are selected on the basis of correlation coefficient $\left(\mathrm{r}^{2}\right)$ and cross-validated correlation coefficient $\left(\mathrm{r}_{\mathrm{cv}}^{2}\right)$ and are presented below:

$$
\log \mathrm{BCF}_{\text {pre }}=7.31267 \times \mathrm{Q} 3+5.86732
$$

$\log \mathrm{BCF}_{\text {pre }}=3.98933 \times \mathrm{Q} 4+6.78289 \times \mathrm{Q} 3+6.25715(2)$ $\log \mathrm{BCF}_{\mathrm{pre}}=2.73959 \times \mathrm{Q} 5+3.83783 \times \mathrm{Q} 4+5.75907 \times$ Q3 +6.48859 
ATOMIC CHARGES AT VARIOUS SITES OF BIPHENYL AND ITS DERIVATIVES AS CALCULATED BY DFT METHODS, EXPERIMENTAL BCF AND PREDICTED BCF

\begin{tabular}{|c|c|c|c|c|c|c|c|c|c|c|c|c|c|c|}
\hline \multirow{2}{*}{ S. No. } & \multicolumn{12}{|c|}{${ }^{\mathrm{b}} \mathrm{Q}$} & \multirow{2}{*}{$\begin{array}{c}{ }^{\mathrm{c}} \log \\
\mathrm{BCF}_{\text {exp }}\end{array}$} & \multirow{2}{*}{$\begin{array}{l}{ }^{\mathrm{d}} \log \\
\mathrm{BCF}_{\mathrm{pre}}\end{array}$} \\
\hline & $\mathrm{Q}_{6}$ & $\mathrm{Q}_{5}$ & $\mathrm{Q}_{4}$ & $\mathrm{Q}_{3}$ & $\mathrm{Q}_{2}$ & $\mathrm{Q}_{1}$ & $\mathrm{Q}_{1}$ & $\mathrm{Q}_{2}$ & $\mathrm{Q}_{3}$, & $\mathrm{Q}_{4}$ & $\mathrm{Q}_{5}$, & $\mathrm{Q}_{6}$ & & \\
\hline 1 & -0.316 & -0.242 & -0.239 & -0.241 & -0.316 & 0.254 & 0.254 & -0.316 & -0.242 & -0.239 & -0.241 & -0.316 & 2.64 & 2.52 \\
\hline 2 & -0.310 & -0.231 & -0.080 & -0.230 & -0.312 & 0.279 & 0.253 & -0.314 & -0.240 & -0.239 & -0.240 & -0.314 & 2.77 & 3.05 \\
\hline 3 & -0.258 & -0.284 & -0.240 & -0.227 & -0.162 & 0.304 & 0.304 & -0.164 & -0.228 & -0.240 & -0.283 & -0.257 & 3.38 & 3.41 \\
\hline 4 & -0.312 & -0.255 & -0.235 & -0.078 & -0.150 & 0.338 & 0.258 & -0.287 & -0.246 & -0.236 & -0.252 & -0.298 & 4.11 & 3.90 \\
\hline $5^{\mathrm{a}}$ & -0.285 & -0.263 & -0.233 & -0.238 & -0.176 & 0.314 & 0.296 & -0.255 & -0.092 & -0.243 & -0.235 & -0.326 & 3.80 & 3.49 \\
\hline 6 & -0.288 & -0.253 & -0.084 & -0.217 & -0.176 & 0.337 & 0.261 & -0.293 & -0.242 & -0.238 & -0.248 & -0.307 & 3.55 & 3.70 \\
\hline 7 & -0.292 & -0.261 & -0.236 & -0.233 & -0.177 & 0.315 & 0.286 & -0.287 & -0.234 & -0.081 & -0.238 & -0.301 & 3.57 & 3.61 \\
\hline 8 & -0.244 & -0.120 & -0.245 & -0.215 & -0.204 & 0.350 & 0.259 & -0.288 & -0.243 & -0.235 & -0.252 & -0.304 & 3.89 & 3.79 \\
\hline 9 & -0.300 & -0.074 & -0.242 & -0.077 & -0.298 & 0.307 & 0.246 & -0.301 & -0.246 & -0.231 & -0.244 & -0.306 & 3.78 & 3.96 \\
\hline 10 & -0.309 & -0.230 & -0.081 & -0.229 & -0.310 & 0.277 & 0.277 & -0.309 & -0.230 & -0.081 & -0.229 & -0.310 & 3.28 & 3.50 \\
\hline 11 & -0.252 & -0.072 & -0.216 & -0.209 & -0.184 & 0.227 & 0.209 & -0.167 & -0.229 & -0.219 & -0.234 & -0.267 & 4.11 & 4.29 \\
\hline 12 & -0.285 & -0.252 & -0.085 & -0.217 & -0.176 & 0.337 & 0.287 & -0.288 & -0.234 & -0.081 & -0.238 & -0.301 & 4.20 & 4.14 \\
\hline 13 & -0.257 & -0.099 & -0.093 & -0.214 & -0.211 & 0.380 & 0.252 & -0.287 & -0.242 & -0.235 & -0.250 & -0.303 & 4.26 & 4.11 \\
\hline 14 & -0.244 & -0.119 & -0.244 & -0.215 & -0.204 & 0.349 & 0.282 & -0.281 & -0.235 & -0.079 & -0.240 & -0.298 & 4.23 & 4.21 \\
\hline 15 & -0.285 & -0.222 & -0.227 & -0.073 & -0.137 & 0.219 & 0.220 & -0.143 & -0.071 & -0.229 & -0.222 & -0.279 & 4.23 & 4.52 \\
\hline 16 & -0.252 & -0.219 & -0.228 & -0.069 & -0.131 & 0.205 & 0.276 & -0.143 & -0.243 & -0.207 & -0.246 & -0.154 & 4.84 & 4.61 \\
\hline 17 & -0.291 & -0.235 & -0.027 & -0.233 & -0.139 & 0.238 & 0.239 & -0.139 & -0.233 & -0.027 & -0.235 & -0.291 & 4.85 & 4.68 \\
\hline 18 & -0.310 & -0.036 & -0.039 & -0.221 & -0.166 & 0.268 & 0.238 & -0.158 & -0.231 & -0.206 & -0.222 & -0.302 & 5.00 & 4.94 \\
\hline $19^{\mathrm{a}}$ & -0.281 & -0.236 & -0.026 & -0.234 & -0.136 & 0.230 & 0.264 & -0.178 & -0.207 & -0.224 & -0.033 & -0.305 & 4.84 & 4.64 \\
\hline 20 & -0.305 & -0.034 & -0.223 & -0.206 & -0.179 & 0.267 & 0.267 & -0.179 & -0.207 & -0.223 & -0.035 & -0.305 & 4.63 & 4.64 \\
\hline 21 & -0.111 & -0.252 & -0.185 & -0.251 & -0.111 & 0.255 & 0.255 & -0.111 & -0.252 & -0.185 & -0.252 & -0.111 & 3.85 & 4.06 \\
\hline 22 & -0.189 & -0.234 & -0.219 & -0.067 & -0.161 & 0.322 & 0.194 & -0.292 & -0.214 & -0.038 & -0.214 & -0.291 & 4.60 & 4.65 \\
\hline $23^{\mathrm{a}}$ & -0.265 & -0.068 & -0.250 & -0.218 & -0.183 & 0.325 & 0.313 & -0.286 & -0.047 & -0.058 & -0.235 & -0.334 & 4.77 & 4.90 \\
\hline 24 & -0.330 & -0.227 & -0.062 & -0.044 & -0.305 & 0.296 & 0.296 & -0.305 & -0.044 & -0.062 & -0.227 & -0.330 & 4.59 & 4.58 \\
\hline 25 & -0.316 & -0.228 & -0.053 & -0.042 & -0.152 & 0.265 & 0.268 & -0.178 & -0.206 & -0.224 & -0.032 & -0.310 & 5.38 & 5.17 \\
\hline 26 & -0.320 & -0.015 & -0.247 & -0.016 & -0.186 & 0.281 & 0.223 & -0.137 & -0.233 & -0.024 & -0.240 & -0.269 & 5.00 & 5.35 \\
\hline 27 & -0.307 & -0.040 & -0.035 & -0.223 & -0.162 & 0.263 & 0.260 & -0.157 & -0.047 & -0.231 & -0.206 & -0.319 & 5.43 & 5.17 \\
\hline $28^{a}$ & -0.310 & -0.037 & -0.038 & -0.221 & -0.167 & 0.269 & 0.236 & -0.140 & -0.234 & -0.024 & -0.236 & -0.286 & 5.00 & 5.23 \\
\hline 29 & -0.266 & -0.037 & -0.038 & -0.216 & -0.172 & 0.240 & 0.217 & -0.147 & -0.229 & -0.028 & -0.230 & -0.252 & 5.40 & 5.29 \\
\hline 30 & -0.309 & -0.256 & -0.052 & -0.064 & -0.150 & 0.341 & 0.305 & -0.288 & -0.045 & -0.062 & -0.236 & -0.321 & 5.00 & 5.23 \\
\hline 31 & -0.168 & -0.262 & -0.030 & -0.080 & -0.146 & 0.310 & 0.207 & -0.297 & -0.017 & -0.239 & -0.186 & -0.312 & 5.00 & 5.06 \\
\hline 32 & -0.326 & -0.040 & -0.039 & -0.227 & -0.210 & 0.306 & 0.245 & -0.319 & -0.020 & -0.056 & -0.203 & -0.350 & 5.00 & 5.02 \\
\hline $33^{\mathrm{a}}$ & -0.345 & -0.024 & -0.091 & -0.027 & -0.345 & 0.338 & 0.276 & -0.291 & -0.048 & -0.056 & -0.233 & -0.321 & 5.81 & 4.93 \\
\hline 34 & -0.317 & -0.227 & -0.052 & -0.043 & -0.148 & 0.262 & 0.261 & -0.149 & -0.044 & -0.052 & -0.227 & -0.316 & 5.77 & 5.50 \\
\hline 35 & -0.138 & -0.232 & -0.213 & -0.066 & -0.107 & 0.279 & 0.279 & -0.108 & -0.067 & -0.213 & -0.231 & -0.137 & 5.43 & 4.99 \\
\hline 36 & -0.329 & -0.027 & -0.068 & -0.020 & -0.186 & 0.283 & 0.222 & -0.136 & -0.234 & -0.025 & -0.237 & -0.269 & 5.88 & 5.76 \\
\hline 37 & -0.280 & -0.230 & -0.053 & -0.045 & -0.146 & 0.236 & 0.235 & -0.159 & -0.223 & -0.035 & -0.040 & -0.273 & 5.39 & 5.51 \\
\hline $38^{\mathrm{a}}$ & -0.339 & -0.028 & -0.068 & -0.015 & -0.194 & 0.296 & 0.261 & -0.179 & -0.205 & -0.222 & -0.036 & -0.298 & 5.81 & 5.65 \\
\hline 39 & -0.287 & -0.011 & -0.245 & -0.017 & -0.165 & 0.266 & 0.244 & -0.104 & -0.261 & -0.006 & -0.262 & -0.111 & 5.39 & 5.29 \\
\hline 40 & -0.168 & -0.042 & -0.241 & -0.042 & -0.165 & 0.319 & 0.234 & -0.157 & -0.209 & -0.217 & -0.035 & -0.251 & 5.54 & 5.45 \\
\hline 41 & -0.305 & -0.042 & -0.036 & -0.221 & -0.169 & 0.267 & 0.267 & -0.169 & -0.221 & -0.036 & -0.042 & -0.304 & 5.65 & 5.51 \\
\hline 42 & -0.091 & -0.259 & -0.008 & -0.260 & -0.091 & 0.242 & 0.244 & -0.091 & -0.259 & -0.007 & -0.260 & -0.092 & 4.93 & 4.98 \\
\hline $43^{a}$ & -0.370 & -0.024 & -0.070 & -0.027 & -0.240 & 0.348 & 0.241 & -0.317 & -0.020 & -0.056 & -0.204 & -0.350 & 5.39 & 5.41 \\
\hline 44 & -0.303 & -0.261 & -0.049 & -0.069 & -0.140 & 0.331 & 0.352 & -0.328 & -0.030 & -0.097 & -0.032 & -0.331 & 5.39 & 5.61 \\
\hline 45 & -0.330 & -0.032 & -0.089 & -0.032 & -0.330 & 0.318 & 0.318 & -0.330 & -0.032 & -0.089 & -0.032 & -0.330 & 5.97 & 5.37 \\
\hline 46 & -0.287 & -0.030 & -0.065 & -0.019 & -0.167 & 0.259 & 0.288 & -0.126 & -0.071 & -0.211 & -0.233 & -0.157 & 5.80 & 5.78 \\
\hline 47 & -0.298 & -0.062 & -0.068 & -0.026 & -0.189 & 0.265 & 0.239 & -0.185 & -0.195 & -0.061 & -0.065 & -0.264 & 5.80 & 5.83 \\
\hline $48^{\mathrm{a}}$ & -0.292 & -0.023 & -0.070 & -0.016 & -0.169 & 0.264 & 0.245 & -0.106 & -0.260 & -0.006 & -0.260 & -0.110 & 5.80 & 5.73 \\
\hline 49 & -0.143 & -0.260 & -0.027 & -0.077 & -0.114 & 0.278 & 0.233 & -0.145 & -0.222 & -0.035 & -0.039 & -0.253 & 5.84 & 5.83 \\
\hline 50 & -0.135 & -0.260 & -0.027 & -0.079 & -0.114 & 0.272 & 0.230 & -0.128 & -0.044 & -0.053 & -0.229 & -0.257 & 5.80 & 5.80 \\
\hline 51 & -0.173 & -0.260 & -0.028 & -0.081 & -0.148 & 0.317 & 0.230 & -0.323 & 0.002 & -0.073 & 0.002 & -0.325 & 5.84 & 5.59 \\
\hline 52 & -0.343 & -0.026 & -0.067 & -0.020 & -0.191 & 0.297 & 0.297 & -0.191 & -0.020 & -0.067 & -0.026 & -0.342 & 5.81 & 6.33 \\
\hline $53^{\mathrm{a}}$ & -0.170 & -0.061 & -0.055 & -0.062 & -0.163 & 0.318 & 0.223 & -0.126 & -0.044 & -0.055 & -0.228 & -0.253 & 5.92 & 6.07 \\
\hline 54 & -0.293 & -0.023 & -0.069 & -0.017 & -0.170 & 0.264 & 0.278 & -0.113 & -0.076 & -0.029 & -0.258 & -0.144 & 5.92 & 6.01 \\
\hline 55 & -0.184 & -0.067 & -0.052 & -0.063 & -0.160 & 0.330 & 0.262 & -0.167 & -0.020 & -0.242 & -0.015 & -0.285 & 5.88 & 6.01 \\
\hline 56 & -0.146 & -0.042 & -0.241 & -0.041 & -0.146 & 0.311 & 0.311 & -0.147 & -0.042 & -0.241 & -0.041 & -0.146 & 5.82 & 5.74 \\
\hline 57 & -0.148 & -0.061 & -0.057 & -0.061 & -0.146 & 0.308 & 0.311 & -0.148 & -0.041 & -0.241 & -0.040 & -0.148 & 5.71 & 6.12 \\
\hline $58^{\mathrm{a}}$ & -0.155 & -0.057 & -0.059 & -0.057 & -0.152 & 0.314 & 0.315 & -0.157 & -0.058 & -0.058 & -0.060 & -0.148 & 5.44 & 6.43 \\
\hline
\end{tabular}




\begin{tabular}{|c|c|c|c|c|c|c|c|c|c|c|c|c|c|c|}
\hline \multirow{3}{*}{ S. No. } & \multicolumn{12}{|c|}{$\begin{array}{l}\text { TABLE-3 } \\
\text { TES OF BIPHENYL AND ITS DERIVATIVES AS } \\
\text { EXPERIMENTAL BCF AND PREDICTED BCF }\end{array}$} & \multirow{3}{*}{$\stackrel{{ }^{\mathrm{c}} \log }{\mathrm{BCF}_{\text {exp }}}$} & \multirow{3}{*}{${ }^{\mathrm{d}} \log$} \\
\hline & \multicolumn{12}{|c|}{${ }^{\mathrm{b}} \rho_{\mathrm{H}}$} & & \\
\hline & $\rho_{6 \mathrm{H}}$ & $\rho_{5 \mathrm{H}}$ & $\rho_{4 \mathrm{H}}$ & $\rho_{3 \mathrm{H}}$ & $\rho_{2 \mathrm{H}}$ & $\rho_{1 \mathrm{H}}$ & $\rho_{I^{\prime} \mathrm{H}}$ & $\rho_{2^{\prime} \cdot \mathrm{H}}$ & $\rho_{3^{\prime} \mathrm{H}}$ & $\rho_{4^{\prime} \mathrm{H}}$ & $\rho_{5^{\prime} \mathrm{H}}$ & $\rho_{6^{\prime} \mathrm{H}}$ & & \\
\hline 1 & 0.082 & 0.024 & 0.164 & 0.024 & 0.082 & 0.124 & 0.124 & 0.082 & 0.024 & 0.164 & 0.024 & 0.082 & 2.64 & 2.92 \\
\hline 2 & 0.068 & 0.032 & 0.142 & 0.033 & 0.068 & 0.125 & 0.103 & 0.073 & 0.020 & 0.143 & 0.019 & 0.074 & 2.77 & 3.54 \\
\hline 3 & 0.012 & 0.063 & 0.080 & 0.005 & 0.116 & 0.105 & 0.104 & 0.115 & 0.004 & 0.080 & 0.062 & 0.012 & 3.38 & 3.48 \\
\hline 4 & 0.096 & 0.011 & 0.145 & 0.024 & 0.049 & 0.098 & 0.141 & 0.069 & 0.038 & 0.171 & 0.020 & 0.088 & 4.11 & 3.70 \\
\hline 5 & 0.043 & 0.024 & 0.109 & 0.009 & 0.079 & 0.099 & 0.102 & 0.015 & 0.070 & 0.174 & 0.005 & 0.159 & 3.80 & 4.04 \\
\hline 6 & 0.053 & 0.033 & 0.118 & 0.021 & 0.074 & 0.114 & 0.120 & 0.070 & 0.028 & 0.154 & 0.020 & 0.079 & 3.55 & 3.41 \\
\hline 7 & 0.050 & 0.022 & 0.111 & 0.009 & 0.081 & 0.087 & 0.137 & 0.061 & 0.043 & 0.145 & 0.034 & 0.067 & 3.57 & 3.96 \\
\hline 8 & 0.014 & 0.054 & 0.127 & 0.004 & 0.141 & 0.093 & 0.116 & 0.065 & 0.027 & 0.145 & 0.022 & 0.070 & 3.89 & 3.39 \\
\hline 9 & 0.074 & 0.019 & 0.171 & 0.018 & 0.077 & 0.091 & 0.140 & 0.079 & 0.028 & 0.172 & 0.029 & 0.077 & 3.78 & 3.72 \\
\hline $10^{\mathrm{a}}$ & 0.062 & 0.028 & 0.126 & 0.028 & 0.062 & 0.106 & 0.106 & 0.062 & 0.028 & 0.126 & 0.028 & 0.062 & 3.28 & 4.34 \\
\hline $11^{\mathrm{a}}$ & 0.047 & 0.173 & 0.068 & 0.054 & 0.183 & 0.070 & 0.013 & 0.010 & 0.000 & 0.009 & 0.006 & 0.002 & 4.11 & 5.06 \\
\hline 12 & 0.049 & 0.026 & 0.102 & 0.018 & 0.065 & 0.091 & 0.123 & 0.059 & 0.038 & 0.134 & 0.031 & 0.063 & 4.20 & 4.31 \\
\hline 13 & 0.028 & 0.047 & 0.120 & 0.009 & 0.098 & 0.102 & 0.112 & 0.066 & 0.026 & 0.143 & 0.020 & 0.071 & 4.26 & 3.83 \\
\hline 14 & 0.021 & 0.035 & 0.111 & 0.003 & 0.112 & 0.073 & 0.127 & 0.055 & 0.040 & 0.133 & 0.035 & 0.058 & 4.23 & 4.32 \\
\hline 15 & 0.068 & 0.062 & 0.005 & 0.082 & 0.072 & 0.003 & 0.003 & 0.089 & 0.100 & 0.006 & 0.076 & 0.084 & 4.23 & 4.32 \\
\hline 16 & 0.152 & 0.129 & 0.012 & 0.175 & 0.154 & 0.005 & 0.001 & 0.004 & 0.008 & 0.000 & 0.008 & 0.005 & 4.84 & 4.65 \\
\hline 17 & 0.017 & 0.069 & 0.088 & 0.007 & 0.064 & 0.119 & 0.119 & 0.064 & 0.008 & 0.089 & 0.069 & 0.018 & 4.85 & 4.70 \\
\hline 18 & 0.006 & 0.134 & 0.098 & 0.006 & 0.129 & 0.109 & 0.051 & 0.048 & 0.002 & 0.041 & 0.033 & 0.006 & 5.00 & 5.03 \\
\hline 19 & 0.008 & 0.017 & 0.030 & 0.005 & 0.019 & 0.038 & 0.073 & 0.172 & 0.040 & 0.073 & 0.149 & 0.029 & 4.84 & 4.97 \\
\hline 20 & 0.011 & 0.085 & 0.054 & 0.018 & 0.110 & 0.052 & 0.051 & 0.107 & 0.017 & 0.053 & 0.082 & 0.011 & 4.63 & 4.84 \\
\hline 21 & 0.065 & 0.112 & 0.003 & 0.112 & 0.065 & 0.002 & 0.001 & 0.062 & 0.106 & 0.003 & 0.106 & 0.062 & 3.85 & 3.88 \\
\hline 22 & 0.061 & 0.002 & 0.054 & 0.025 & 0.008 & 0.049 & 0.182 & 0.047 & 0.058 & 0.154 & 0.057 & 0.047 & 4.60 & 4.95 \\
\hline 23 & 0.015 & 0.036 & 0.102 & 0.003 & 0.106 & 0.071 & 0.105 & 0.025 & 0.057 & 0.139 & 0.017 & 0.086 & 4.77 & 4.79 \\
\hline $24^{\mathrm{a}}$ & 0.076 & 0.017 & 0.127 & 0.038 & 0.037 & 0.092 & 0.092 & 0.037 & 0.038 & 0.127 & 0.017 & 0.076 & 4.59 & 5.36 \\
\hline 25 & 0.017 & 0.007 & 0.031 & 0.011 & 0.008 & 0.029 & 0.073 & 0.174 & 0.041 & 0.072 & 0.151 & 0.030 & 5.38 & 5.26 \\
\hline 26 & 0.073 & 0.141 & 0.027 & 0.074 & 0.187 & 0.040 & 0.022 & 0.009 & 0.003 & 0.016 & 0.009 & 0.004 & 5.00 & 5.09 \\
\hline 27 & 0.011 & 0.159 & 0.100 & 0.010 & 0.143 & 0.113 & 0.026 & 0.008 & 0.007 & 0.031 & 0.004 & 0.022 & 5.43 & 5.48 \\
\hline 28 & 0.004 & 0.116 & 0.093 & 0.004 & 0.115 & 0.101 & 0.073 & 0.037 & 0.007 & 0.055 & 0.037 & 0.013 & 5.00 & 5.39 \\
\hline 29 & 0.020 & 0.169 & 0.095 & 0.012 & 0.146 & 0.106 & 0.021 & 0.009 & 0.002 & 0.013 & 0.009 & 0.004 & 5.40 & 5.74 \\
\hline 30 & 0.059 & 0.016 & 0.102 & 0.024 & 0.040 & 0.081 & 0.103 & 0.026 & 0.056 & 0.138 & 0.014 & 0.089 & 5.00 & 5.30 \\
\hline 31 & 0.038 & 0.001 & 0.033 & 0.031 & 0.002 & 0.038 & 0.071 & 0.026 & 0.145 & 0.117 & 0.017 & 0.199 & 5.00 & 4.82 \\
\hline 32 & 0.007 & 0.067 & 0.086 & 0.002 & 0.085 & 0.082 & 0.098 & 0.019 & 0.044 & 0.114 & 0.022 & 0.061 & 5.00 & 5.34 \\
\hline 33 & 0.056 & 0.022 & 0.125 & 0.026 & 0.050 & 0.080 & 0.099 & 0.034 & 0.042 & 0.131 & 0.019 & 0.076 & 5.81 & 5.51 \\
\hline 34 & 0.033 & 0.048 & 0.102 & 0.019 & 0.036 & 0.115 & 0.115 & 0.036 & 0.019 & 0.102 & 0.048 & 0.033 & 5.77 & 5.48 \\
\hline 35 & 0.079 & 0.057 & 0.014 & 0.100 & 0.048 & 0.009 & 0.008 & 0.045 & 0.092 & 0.013 & 0.053 & 0.073 & 5.43 & 5.36 \\
\hline 36 & 0.012 & 0.122 & 0.067 & 0.011 & 0.126 & 0.082 & 0.071 & 0.033 & 0.005 & 0.050 & 0.042 & 0.008 & 5.88 & 5.50 \\
\hline $37^{\mathrm{a}}$ & 0.001 & 0.001 & 0.001 & 0.000 & 0.002 & 0.006 & 0.107 & 0.151 & 0.016 & 0.095 & 0.183 & 0.026 & 5.39 & 6.65 \\
\hline 38 & 0.003 & 0.012 & 0.022 & 0.002 & 0.016 & 0.022 & 0.069 & 0.174 & 0.045 & 0.069 & 0.156 & 0.039 & 5.81 & 5.38 \\
\hline 39 & 0.107 & 0.150 & 0.018 & 0.091 & 0.189 & 0.030 & 0.000 & 0.001 & 0.001 & 0.000 & 0.001 & 0.001 & 5.39 & 5.32 \\
\hline 40 & 0.005 & 0.005 & 0.000 & 0.005 & 0.006 & 0.001 & 0.064 & 0.176 & 0.056 & 0.062 & 0.173 & 0.060 & 5.54 & 5.70 \\
\hline 41 & 0.003 & 0.079 & 0.072 & 0.002 & 0.082 & 0.078 & 0.077 & 0.081 & 0.002 & 0.071 & 0.078 & 0.003 & 5.65 & 5.71 \\
\hline 42 & 0.027 & 0.029 & 0.077 & 0.039 & 0.022 & 0.141 & 0.142 & 0.023 & 0.037 & 0.078 & 0.031 & 0.027 & 4.93 & 4.62 \\
\hline 43 & 0.011 & 0.049 & 0.082 & 0.003 & 0.067 & 0.072 & 0.112 & 0.018 & 0.052 & 0.129 & 0.026 & 0.067 & 5.39 & 5.50 \\
\hline 44 & 0.060 & 0.019 & 0.108 & 0.028 & 0.038 & 0.090 & 0.093 & 0.041 & 0.039 & 0.137 & 0.021 & 0.065 & 5.39 & 5.28 \\
\hline 45 & 0.051 & 0.027 & 0.130 & 0.027 & 0.051 & 0.086 & 0.086 & 0.051 & 0.027 & 0.130 & 0.027 & 0.051 & 5.97 & 5.50 \\
\hline 46 & 0.039 & 0.107 & 0.027 & 0.028 & 0.106 & 0.039 & 0.007 & 0.039 & 0.073 & 0.010 & 0.043 & 0.058 & 5.80 & 5.45 \\
\hline 47 & 0.002 & 0.038 & 0.044 & 0.001 & 0.044 & 0.048 & 0.097 & 0.114 & 0.005 & 0.088 & 0.125 & 0.007 & 5.80 & 6.11 \\
\hline 48 & 0.063 & 0.174 & 0.046 & 0.043 & 0.176 & 0.066 & 0.001 & 0.002 & 0.001 & 0.000 & 0.002 & 0.001 & 5.80 & 5.64 \\
\hline $49^{\mathrm{a}}$ & 0.001 & 0.001 & 0.001 & 0.000 & 0.002 & 0.005 & 0.108 & 0.149 & 0.015 & 0.095 & 0.182 & 0.027 & 5.84 & 6.73 \\
\hline 50 & 0.140 & 0.047 & 0.055 & 0.191 & 0.052 & 0.049 & 0.003 & 0.007 & 0.010 & 0.001 & 0.006 & 0.008 & 5.80 & 5.97 \\
\hline 51 & 0.052 & 0.003 & 0.057 & 0.040 & 0.005 & 0.067 & 0.121 & 0.033 & 0.045 & 0.148 & 0.043 & 0.034 & 5.84 & 5.66 \\
\hline 52 & 0.004 & 0.081 & 0.062 & 0.003 & 0.086 & 0.069 & 0.069 & 0.084 & 0.003 & 0.062 & 0.079 & 0.003 & 5.81 & 5.83 \\
\hline 53 & 0.125 & 0.127 & 0.004 & 0.128 & 0.125 & 0.003 & 0.000 & 0.005 & 0.009 & 0.001 & 0.004 & 0.007 & 5.92 & 6.07 \\
\hline 54 & 0.061 & 0.172 & 0.048 & 0.040 & 0.175 & 0.067 & 0.002 & 0.003 & 0.001 & 0.000 & 0.002 & 0.001 & 5.92 & 5.69 \\
\hline 55 & 0.001 & 0.004 & 0.001 & 0.001 & 0.004 & 0.001 & 0.030 & 0.187 & 0.088 & 0.018 & 0.147 & 0.105 & 5.88 & 5.56 \\
\hline 56 & 0.067 & 0.068 & 0.002 & 0.068 & 0.067 & 0.002 & 0.002 & 0.067 & 0.068 & 0.002 & 0.068 & 0.067 & 5.82 & 6.00 \\
\hline 57 & 0.009 & 0.010 & 0.000 & 0.010 & 0.009 & 0.000 & 0.003 & 0.126 & 0.124 & 0.003 & 0.124 & 0.126 & 5.71 & 6.06 \\
\hline $58^{\mathrm{a}}$ & 0.067 & 0.063 & 0.002 & 0.068 & 0.062 & 0.002 & 0.002 & 0.065 & 0.071 & 0.002 & 0.066 & 0.070 & 5.44 & 6.10 \\
\hline
\end{tabular}

${ }^{\mathrm{a}}$ Data point not used in the model, ${ }^{\mathrm{b}} \rho_{\mathrm{H}} \mathrm{HOMO}$ density of the carbon atom at a respective site of the molecule, ${ }^{\mathrm{c}}$ Experimental logarithmic BCF values as taken from literature [24], ${ }^{\mathrm{d}}$ Predicted logarithmic BCF values by eqn. 22. 
$\log \mathrm{BCF}_{\mathrm{pre}}=3.68829 \times \mathrm{Q} 5+3.6534 \times \mathrm{Q} 4+5.20816 \times$ $\mathrm{Q} 3+5.51755 \times \mathrm{Q} 2+7.52158$

$\log \mathrm{BCF}_{\text {pre }}=3.58483 \times \mathrm{Q} 5+3.4302 \times \mathrm{Q} 4+4.6663 \times$ $\mathrm{Q} 3+5.57053 \times \mathrm{Q} 2+1.44939 \times \mathrm{Q}^{\prime}+7.6443$

$\log \mathrm{BCF}_{\text {pre }}=2.26451 \times \mathrm{Q} 6+4.02966 \times \mathrm{Q} 5+3.30571 \times$ $\mathrm{Q} 4+4.93578 \times \mathrm{Q} 3+3.97865 \times \mathrm{Q} 2+1.29065 \times$ Q4' +7.97909

$\log \mathrm{BCF}_{\mathrm{pre}}=1.65056 \times \mathrm{Q} 6+3.82019 \times \mathrm{Q} 5+3.56547 \times$ $\mathrm{Q} 4+4.6762 \times \mathrm{Q} 3+4.32587 \times \mathrm{Q} 2+0.425289 \times$

$\mathrm{Q} 1+1.2721 \times \mathrm{Q}^{\prime}+7.7787$

$\log \mathrm{BCF}_{\text {pre }}=4.37131 \times \mathrm{Q} 6+4.54443 \times \mathrm{Q} 5+3.85701 \times$ $\mathrm{Q} 4+5.57252 \times \mathrm{Q} 3+4.65045 \times \mathrm{Q} 2-3.36609 \times \mathrm{Q} 1$

$+5.8007 \times \mathrm{Q}^{\prime}{ }^{\prime}-3.55156 \times \mathrm{Q} 6{ }^{\prime}+7.21326$

$\log \mathrm{BCF}_{\text {pre }}=4.38018 \times \mathrm{Q} 6+4.61634 \times \mathrm{Q} 5+3.86303 \times$ $\mathrm{Q} 4+5.58354 \times \mathrm{Q} 3+4.82281 \times \mathrm{Q} 2-3.59199 \times \mathrm{Q} 1$ $+5.81215 \times \mathrm{Q}^{\prime}{ }^{\prime}-0.274077 \times \mathrm{Q}^{\prime}{ }^{\prime}-3.46388 \times \mathrm{Q}^{\prime}+$ 7.2887

$\log \mathrm{BCF}_{\mathrm{pre}}=2.70639 \times \mathrm{Q} 5+2.36757 \times \mathrm{Q} 4+3.42427 \times$ $\mathrm{Q} 3+3.69139 \times \mathrm{Q} 2+1.65867 \times \mathrm{Q} 1+0.706296 \times \mathrm{Q}^{\prime}$ $+2.07852 \times \mathrm{Q}^{\prime}+1.86941 \times \mathrm{Q}^{\prime}+1.98578 \times \mathrm{Q}^{\prime}{ }^{\prime}+$ $1.96214 \times \mathrm{Q}^{\prime}+7.34506$

$\log \mathrm{BCF}_{\mathrm{pre}}=4.91357 \times \mathrm{Q} 6+4.57264 \times \mathrm{Q} 5+3.20577 \times$

$\mathrm{Q} 4+5.33602 \times \mathrm{Q} 3+4.48698 \times \mathrm{Q} 2-3.71172 \times \mathrm{Q} 1$

$+6.74472 \times \mathrm{Q}^{\prime}+0.0458702 \times \mathrm{Q}^{\prime}+0.121179 \times \mathrm{Q}^{\prime}$

$+1.77042 \times \mathrm{Q}^{\prime}{ }^{\prime}-3.88007 \times \mathrm{Q}^{\prime}+7.25792$

$\log \mathrm{BCF}_{\text {pre }}=4.01452 \times \mathrm{Q} 6+4.19279 \times \mathrm{Q} 5+3.0018 \times$

$\mathrm{Q} 4+4.85451 \times \mathrm{Q} 3+4.18059 \times \mathrm{Q} 2-2.61371 \times \mathrm{Q} 1$

$+5.39416 \times \mathrm{Q}^{\prime}+0.421213 \times \mathrm{Q}^{\prime}+0.487842 \times$

$\mathrm{Q}^{\prime}+1.86228 \times \mathrm{Q}^{\prime}+0.57685 \times \mathrm{Q}^{\prime}{ }^{\prime}-2.95444 \times$

Q6' +7.35851

The regression summary of these equations has also been presented in Table-4. A close look at this table indicates that the reliable model is the eqn. 7 as it has highest value of ' $\mathrm{r}^{2}$, and lowest value of 's' among the rest equations. All the descriptors of this model have positive coefficient values indicating that these have direct relationship with the bioconcentration factors, except $\mathrm{Q}_{1}$ and $\mathrm{Q}_{6}$, which have a negative coefficient values indicating that these have inverse relationship with bioconcentration factors. Predicted bioconcentration factor as obtained by this equation has also been presented in Table- 2 . The predicted bioconcentration factor is reliable as it is evident from its standard error value (0.27).

TABLE-4

REGRESSION SUMMARY OF MODELS DERIVED FROM ATOMIC CHARGES AS INDEPENDENT VARIABLES

\begin{tabular}{cccccc}
\hline Eqn. No. & $\mathrm{k}$ & $\mathrm{n}$ & $\mathrm{r}^{2}$ & $\mathrm{r}_{\mathrm{CV}}$ & $\mathrm{s}$ \\
\hline 1 & 1 & 49 & 0.645 & 0.639 & 0.482 \\
2 & 2 & 49 & 0.814 & 0.793 & 0.355 \\
3 & 3 & 49 & 0.878 & 0.869 & 0.292 \\
4 & 4 & 56 & 0.900 & 0.874 & 0.288 \\
5 & 5 & 56 & 0.910 & 0.886 & 0.275 \\
6 & 6 & 56 & 0.914 & 0.887 & 0.273 \\
7 & 7 & 56 & 0.917 & 0.879 & 0.270 \\
8 & 8 & 57 & 0.908 & 0.845 & 0.288 \\
9 & 9 & 57 & 0.909 & 0.843 & 0.291 \\
10 & 10 & 57 & 0.911 & 0.832 & 0.292 \\
11 & 11 & 58 & 0.914 & 0.839 & 0.288 \\
12 & 12 & 58 & 0.916 & 0.834 & 0.288 \\
\hline
\end{tabular}

HOMO density $\left(\rho \mathrm{HOMO}=\rho_{\mathrm{H}}\right)$ : FMO theory predicts that the preferable electrophilic reaction in a molecule will take place at the site, where the relative density of the HOMO is high [45]. The HOMO density of each atom of all the polychlorinated biphenyls were calculated by DFT method and the result are reported in Table-3. Similar to partial atomic charges, MLR analysis [42] has also been made by same software using HOMO densities of 12 sites in various combinations. The best twelve models, for $\mathrm{k}=1,2,3,4,5,6,7,8,9,10,11$ and 12 (eqns. 13-24) are presented below:

$$
\begin{aligned}
& \log \mathrm{BCF}_{\text {pre }}=-11.0528 \times \rho_{4 \mathrm{H}}+5.66552 \\
& \log \mathrm{BCF}_{\text {pre }}=-12.4484 \times \rho_{4 \mathrm{H}}-5.57528 \times \rho_{3^{\prime} \mathrm{H}} \\
& +5.96252
\end{aligned}
$$

$\log \mathrm{BCF}_{\text {pre }}=-16.0072 \times \rho_{4 \mathrm{H}}-7.84311 \times \rho_{3 \mathrm{H}}-6.61487 \times$ $\rho_{2}{ }^{\prime}+6.65743$

$\log \mathrm{BCF}_{\text {pre }}=-9.90346 \times \rho_{6 \mathrm{H}}-1.81391 \times \rho_{5 \mathrm{H}}-14.4676 \times$ $\rho_{4 \mathrm{H}}-7.98262 \times \rho_{2^{\prime} \mathrm{H}}+6.89501$

$\log \mathrm{BCF}_{\mathrm{pre}}=-6.84855 \times \rho_{3 \mathrm{H}}-8.00967 \times \rho_{2 \mathrm{H}}-4.06683 \times$ $\rho_{1 \mathrm{H}}-10.6476 \times \rho_{1^{\prime} \mathrm{H}}-9.67037 \times \rho_{6^{\prime} \mathrm{H}}+7.27363$

$\log \mathrm{BCF}_{\text {pre }}=-18.5163 \times \rho_{3 \mathrm{H}}-20.1669 \times \rho_{2 \mathrm{H}}-16.1387 \times$ $\rho_{1 \mathrm{H}}-16.802 \times \rho_{1^{\prime} \mathrm{H}}-17.9421 \times \rho_{2^{\prime} \mathrm{H}}-22.4239 \times \rho_{3^{\prime} \mathrm{H}}+$ 11.264

$\log \mathrm{BCF}_{\mathrm{pre}}=-18.5657 \times \rho_{3 \mathrm{H}}-19.515 \times \rho_{2 \mathrm{H}}-14.6725 \times$ $\rho_{1 \mathrm{H}}-15.8494 \times \rho_{1^{\prime} \mathrm{H}}-17.6719 \times \rho_{2^{\prime} \mathrm{H}}-15.3895 \times \rho_{3^{\prime} \mathrm{H}}$ $-5.63894 \times \rho_{6}{ }^{\prime} \mathrm{H}+11.0543$

$\log \mathrm{BCF}_{\mathrm{pre}}=-21.2936 \times \rho_{3 \mathrm{H}}-22.650 \times \rho_{2 \mathrm{H}}-20.9979 \times$ $\rho_{1 \mathrm{H}}-8.16519 \times \rho_{1^{\prime} \mathrm{H}}-12.2259 \times \rho_{2^{\prime} \mathrm{H}}-23.1899 \times$ $\rho_{3^{\prime} \mathrm{H}}-8.84995 \times \rho_{4^{\prime} \mathrm{H}}-11.1331 \times \rho_{5^{\prime} \mathrm{H}}+12.1834$

$\log \mathrm{BCF}_{\text {pre }}=-15.3068 \times \rho_{5 \mathrm{H}}-3.07892 \times \rho_{4 \mathrm{H}}-21.8963 \times$ $\rho_{3 \mathrm{H}}-11.4376 \times \rho_{2 \mathrm{H}}-10.5214 \times \rho_{1 \mathrm{H}}-22.539 \times \rho_{1^{\prime} \mathrm{H}}$ $-21.2722 \times \rho_{2^{\prime} \mathrm{H}}-11.2643 \times \rho_{3^{\prime} \mathrm{H}}-12.9504 \times \rho_{6^{\prime} \mathrm{H}}$ $+12.318$

$\log \mathrm{BCF}_{\mathrm{pre}}=-5.77578 \times \rho_{6 \mathrm{H}}-15.3989 \times \rho_{5 \mathrm{H}}-1.76363 \times$ $\rho_{4 \mathrm{H}}-17.1514 \times \rho_{3 \mathrm{H}}-11.205 \times \rho_{2 \mathrm{H}}-11.8282 \times \rho_{1 \mathrm{H}}$

$-21.9404 \times \rho_{1^{\prime} \mathrm{H}}-21.613 \times \rho_{2^{\prime} \mathrm{H}}-12.7592 \times \rho_{3^{\prime} \mathrm{H}}$

$-11.5088 \times \rho_{6}{ }^{\prime} \mathrm{H}+12.3434$

$\log \mathrm{BCF}_{\text {pre }}=-12.7279 \times \rho_{6 \mathrm{H}}-5.95064 \times \rho_{5 \mathrm{H}}+3.019 \times$ $\rho_{4 \mathrm{H}}-13.0376 \times \rho_{3 \mathrm{H}}-20.0458 \times \rho_{2 \mathrm{H}}-24.851 \times \rho_{1 \mathrm{H}}$ $-8.06609 \times \rho_{1^{\prime} \mathrm{H}}-12.8066 \times \rho_{2^{\prime} \mathrm{H}}-25.1578 \times \rho_{3^{\prime} \mathrm{H}}$ $-10.5185 \times \rho_{4^{\prime} \mathrm{H}}-14.2126 \times \rho_{5^{\prime} \mathrm{H}}+13.0287$

$\log \mathrm{BCF}_{\mathrm{pre}}=-11.2483 \times \rho_{6 \mathrm{H}}-9.69523 \times \rho_{5 \mathrm{H}}+0.806812$ $\times \rho_{4 \mathrm{H}}-15.1178 \times \rho_{3 \mathrm{H}}-17.7574 \times \rho_{2 \mathrm{H}}-21.4003 \times \rho_{1 \mathrm{H}}$ $-13.8613 \times \rho_{1^{\prime} \mathrm{H}}-12.9165 \times \rho_{2^{\prime} \mathrm{H}}-17.2176 \times \rho_{3^{\prime} \mathrm{H}}$ $-5.65047 \times \rho_{4^{\prime} \mathrm{H}}-14.4009 \times \rho_{5^{\prime} \mathrm{H}}-8.67902 \times \rho_{6^{\prime} \mathrm{H}}$ $+13.2$

The regression summary of these equations has also been presented in Table-5, which indicates that the most reliable model is the eqn. 22 as it has highest value of ' $\mathrm{r}^{2}$ ' and lowest value of 's' among the rest equations. All the descriptors of this model have negative coefficient values, indicating that these have inverse relationship with bioconcentration factors, except $\rho_{4 \mathrm{H}}$, which has a positive coefficient values indicating that it has a direct relationship with the bioconcentration factors. Predicted bioconcentration factor as obtained by this equation has also been presented in Table-3. The predicted 
3. S.E. Bysshe, eds.: W.J. Lyman, W.F. Reehl and D.H. Rosenblatt, Biocon-

TABLE-5

REGRESSION SUMMARY OF MODELS DERIVED FROM HOMO DENSITIES AS INDEPENDENT VARIABLES

\begin{tabular}{cccccc}
\hline Eqn. No. & $\mathrm{k}$ & $\mathrm{n}$ & $\mathrm{r}^{2}$ & $\mathrm{r}_{\mathrm{CV}}^{2}$ & $\mathrm{~s}$ \\
\hline 13 & 1 & 48 & 0.638 & 0.614 & 0.532 \\
14 & 2 & 48 & 0.763 & 0.747 & 0.443 \\
15 & 3 & 48 & 0.825 & 0.811 & 0.386 \\
16 & 4 & 48 & 0.826 & 0.807 & 0.389 \\
17 & 5 & 48 & 0.830 & 0.797 & 0.385 \\
18 & 6 & 50 & 0.825 & 0.754 & 0.396 \\
19 & 7 & 48 & 0.832 & 0.775 & 0.396 \\
20 & 8 & 48 & 0.867 & 0.802 & 0.335 \\
21 & 9 & 53 & 0.865 & 0.749 & 0.346 \\
22 & 10 & 52 & 0.902 & 0.837 & 0.307 \\
23 & 11 & 56 & 0.865 & 0.765 & 0.353 \\
24 & 12 & 58 & 0.840 & 0.693 & 0.397 \\
\hline
\end{tabular}

bioconcentration factor is reliable as it is evident from its 'standard error value (0.307).

On comparing the above eqns. ( 7 and 22 ), we have found that model derived from partial atomic charges has more reliable predicting power than the model derived from $\mathrm{HOMO}$ densities, because the formal model has higher values of $r^{2}$, $\mathrm{r}_{\mathrm{CV}}^{2}$ and lower value of standard error and used less descriptor than the later model. The current study accounts for the previous findings with advanced approaches (DFT) but in more easier and convenient way. Ivanciuc et al. [24] for the first time made QSAR study on these polychlorinated biphenyls aggregated from various literature reports. Predictive ability of their models was ' $r$ ' between 0.903 and 0.935 for splinoid QSSAR and ' $r$ ' between 0.745 and 0.887 for cluster-expansion [24]. In continuation to this Katritzky et al. [20], reported QSAR results of the same data set based on 486 constitutional, topological, geometrical, electrostatic, quantum chemical and thermodynamic descriptors derived solely from molecular structure and calculated using CODESSA Pro software and proposed that two-parameter model satisfactorily describes the relationship between observed and calculated values in terms of statistical parameters. Borges de Melo [46] reported QSAR results based on E-state and topological descriptors using SMILE software and the best model presented five descriptors (one E-state index and four topological descriptors). It has been proven that local atomic charges are important in many chemical reactions and physico-chemical properties of compounds, thus it may also helpful in determining the new methodology of the degradation of polychlorinated biphenyls, which is also a great challenge because these compounds are strongly resistant to destruction due to their high chemical stability [5].

\section{ACKNOWLEDGEMENTS}

The authors are thankful to The Principal, M.L.K. (P.G.) College, Balrampur, India for laboratory facilities and Mr. Harsh Sahu for computational help.

\section{REFERENCES}

1. Ecological Effects Test Guidelines: Fish BCF, EPA/712-C-96-127, US Environmental Protection Agency, Washington, DC (1996).

2. Guidelines for the Identification of PCBs and Materials Containing PCBs, United Nations Environment Programme, First Issue, August (1999) centration Factor in Aquatic Organisms, In: Handbook of Chemical Property Estimation Methods, McGraw Hill, New York (1982).

4. G. Schüürmann and W. Klein, Chemosphere, 17, 1551 (1988); https://doi.org/10.1016/0045-6535(88)90207-X.

5. I. Grabowska, Pol. J. Environ. Stud., 19, 7 (2010).

6. V.K. Sahu and R.K. Singh, Clean-Soil Air Water, 37, 850 (2009); https://doi.org/10.1002/clen.200900170.

7. R.F. Herrick, D.J. Lefkowitz and G.A. Weymouth, Environ. Health Perspect., 115, 173 (2006);

https://doi.org/10.1289/ehp.9646.

8. S.K. Sahu, P.Y. Ajmal, G.G. Pandit and V.D. Puranik, J. Hazard. Mater., 164, 1573 (2009); https://doi.org/10.1016/j.jhazmat.2008.08.113.

9. K. Senthilkumar, K. Kannan, A. Subramanian and S. Tanabe, Environ. Sci. Pollut. Res. Int., 8, 35 (2001); https://doi.org/10.1007/BF02987293.

10. The Role of Bioaccumulation in Environmental Risk Assessment: The Aquatic Environment and Related Food Webs, Technical Report 67, European Centre for Ecotoxicology and Toxicology of Chemicals (ECETOC), Brussels, Belgium (1996).

11. B. Hope, S. Scatolini and E. Titus, Chemosphere, 36, 1247 (1998); https://doi.org/10.1016/S0045-6535(97)10045-5.

12. T. Kunisue, M. Watanabe, A. Subramanian, A.M. Titenko and S. Tanabe, Arch. Environ. Contam. Toxicol., 45, 547 (2003); https://doi.org/10.1007/s00244-003-2213-8.

13. G.M. Swanson, H.E. Ratcliffe and L.J. Fischer, Regul. Toxicol. Pharmacol., 21, 136 (1995); https://doi.org/10.1006/rtph.1995.1018.

14. G. Schade and B. Heinzow, Sci. Total Environ., 215, 31 (1998); https://doi.org/10.1016/S0048-9697(98)00008-4.

15. O. Faroon, D. Jones and C. De Rosa, Toxicol. Ind. Health, 16, 305 (2000); https://doi.org/10.1177/074823370001600708.

16. G. Devanathan, A. Subramanian, M. Someya, A. Sudaryanto, T. Isobe, S. Takahashi, P. Chakraborty and S. Tanabe, Environ. Pollut., 157, 148 (2009);

https://doi.org/10.1016/j.envpol.2008.07.011.

17. G. Devanathan, T. Isobe, A. Subramanian, K.A. Asante, S. Natrajan, P. Palanlappan, S. Takahashi and S. Tanabe, eds.: M. Kawaguchi, K. Misaki, H. Sato, T. Yokokawa, T. Itai, T. M. Nguyen, J. Ono and S. Tanabe, Contamination Status of Polychlorinated Biphenyls and Brominated Flame Retardants in Environmental and Biota Samples from India, In: Interdisciplinary Studies on Environmental Chemistry-Environmental Pollution and Ecotoxicology, pp. 269-277 (2012).

18. I. Cok, A. Bilgili, M. Ozdemir, H. Ozbek, N. Bilgili and S. Burgaz, Bull. Environ. Contam. Toxicol., 59, 577 (1997); https://doi.org/10.1007/s001289900518.

19. C. Hansch and A. Leo, Exploring QSAR: Fundamentals and Applications in Chemistry and Biology, American Chemical Society, Washington, DC (1995).

20. A.R. Katritzky, M. Radzvilovits, S. Slavov, K. Kasemets, K. Tämm and M. Karelson, Toxicol. Environ. Chem., 92, 1233 (2010); https://doi.org/10.1080/02772240903306417.

21. L.T. Qin, S.S. Liu, H.L. Liu and H.L. Ge, Chemosphere, 70, 1577 (2008); https://doi.org/10.1016/j.chemosphere.2007.08.009.

22. C. Mori, H. Fukata, K. Sakurai, T. Jotaki, E. Todaka and Y. Saito, Chemosphere, 73, S235 (2008); https://doi.org/10.1016/j.chemosphere.2007.12.038.

23. D.G. Wang, M. Yang, H.L. Jia, L. Zhou and Y.F. Li, Chemosphere, 73, 38 (2008);

https://doi.org/10.1016/j.chemosphere.2008.05.055.

24. T. Ivanciuc, O. Ivanciuc and D.J. Klein, Mol. Divers., 10, 133 (2006); https://doi.org/10.1007/s11030-005-9003-3.

25. P. Gramatica and E. Papa, QSAR Comb. Sci., 24, 953 (2005); https://doi.org/10.1002/qsar.200530123.

26. J.C. Dearden and N.M. Shinnawei, SAR QSAR Environ. Res., 15, 449 (2004); https://doi.org/10.1080/10629360412331297489.

27. M.T. Sacan, S.S. Erdem, G.A. Ozpinar and I.A. Balcioglu, J. Chem. Inf. Comput. Sci., 44, 985 (2004); https://doi.org/10.1021/ci0342167.

28. P. Gramatica and E. Papa, QSAR Comb. Sci., 22, 374 (2003); https://doi.org/10.1002/qsar.200390027. 
29. P.V. Khadikar, S. Singh, D. Mandloi, S. Joshi and A.V. Bajaj, Bioorg. Med. Chem., 11, 5045 (2003); https://doi.org/10.1016/j.bmc.2003.08.028

30. S. Tao, H. Hu, X. Lu, R.W. Dawson and F. Xu, Chemosphere, 41, 1563 (2000);

https://doi.org/10.1016/S0045-6535(00)00049-7.

31. J. Devillers, S. Bintein and D. Domine, Chemosphere, 33, 1047 (1996); https://doi.org/10.1016/0045-6535(96)00246-9.

32. S. Bintein, J. Devillers and W. Karcher, SAR QSAR Environ. Res., 1, 29 (1993);

https://doi.org/10.1080/10629369308028814.

33. A. Sabljic, H. Guesten, J. Hermens and A. Opperhuizen, Environ. Sci. Technol., 27, 1394 (1993); https://doi.org/10.1021/es00044a015.

34. R.P. Davies and A.J. Dobbs, Water Res., 18, 1253 (1984); https://doi.org/10.1016/0043-1354(84)90030-7.

35. D. Mackay, Environ. Sci. Technol., 16, 274 (1982); https://doi.org/10.1021/es00099a008.

36. M. Karelson, V.S. Lobanov and A.R. Katritzky, Chem. Rev., 96, 1027 (1996); https://doi.org/10.1021/cr950202r.

37. R.G. Parr and W. Yang, Density Functional Theory of Atoms and Molecules, Oxford University Press, New York (1989).
38. P. Geerlings, F.D. Proft and J.M.L. Martin, ed.: S. Seminario, Recent Developments in Density Functional Theory (Theoretical and Computational Chemistry) Elsevier: Amsterdam (1996).

39. K. Singhal, V.K. Sahu, P. Singh and P. Raj, Med. Chem. Res., 23, 1758 (2014);

https://doi.org/10.1007/s00044-013-0752-8

40. A.R. Leach, Molecular Modelling. Principle and Applications, Pearson Education Limited, Harlow, edn 2 (2001).

41. F.A. Pasha, H.K. Srivastava and P.P. Singh, Bioorg. Med. Chem., 13, 6823 (2005); https://doi.org/10.1016/j.bmc.2005.07.064.

42. R.L. Mason, R.F. Gunst and J.T. Webster, Commun. Stat., 4, 277 (1975); https://doi.org/10.1080/03610917508548355.

43. O.P. Agarwal, Polynuclear Aromatic Hydrocarbon in Chemistry of Organic Natural Product, Goel Publishing House, Delhi, India, vol. 1 (2002).

44. G. Klopman, J. Am. Chem. Soc., 90, 223 (1968); https://doi.org/10.1021/ja01004a002.

45. R.G. Parr and W. Yang, J. Am. Chem. Soc., 106, 4049 (1984); https://doi.org/10.1021/ja00326a036.

46. E.B. de Melo, Ecotoxicol. Environ. Saf., 75, 213 (2012); https://doi.org/10.1016/j.ecoenv.2011.08.026 